\title{
Stimulus generalization of response-position in the rat'
}

LEWIS R. GOLLUB

UNIVERSITY OF MARYLAND

Three rats were trained by food reinforcement to poke the ir noses into each of two 1-1/8 in. segments of a 10-in. slot. Two auditory click frequencies signalled whether a response in one segment or the other would be reinforced. Stimulus generalization gradients of location were then obtained in the presence of 4 click frequencies. Although the mean location varied continuously with stimulus value, responding tended to occur primarily around the 2 previously reinforced locations, according to bimodal distributions, with peaks of varying relative heights.

Several recent studies of stimulus generalization have emphasized the importance of a fine-grained analysis of the responding to each stimulus in addition to a simple mean response measure. In a single-stimulus and single-key experiment, Blough (1963) showed that stimuli have varying degrees of control over inter-response times of different value during generalization. Averaging all IRTs together as the dependent variable, as in a response rate measure, therefore obscures this differential control, and produces a degree of averaging artifact.

An explicit way of examining this relationship is to study the stimulus generalization of a continuously variable response, two values of which have been brought under discriminative control during training. In this type of study, each of two responses is reinforced in the presence of one of two stimuli. Experiments have been performed in which humans hummed tones (Cross \& Lane, 1962), rats paused between pressing two bars (Migler, 1964), and pigeons pecked different places on a long strip (Cumming \& Eckerman, 1965). All of these studies showed that responses to intermediate stimuli in the generalization test do not tend to take on intermediate values to those established in training, but rather occur bimodally, with differing frequencies of the two original response distributions. (The mean values of these measures do, however, have intermediate values.) The present experiment extends work in this area by investigating the control of response position in the rat.

\section{Method}

Three male adult, albino rats, maintained at 80 percent of their ad lib weights at about 150 days of age (256, 274, and $275 \mathrm{gm}$ each) were Ss. The experimental space consisted of a box $9 \times 10 \times 8$ in. high, with metal walls, a grid floor, and a plastic top. The box was in a sound-proofed container. In one wall of the box was a slot giving access to a recessed chamber, 1-1/2 in. high, $10 \mathrm{in.} \mathrm{long,} \mathrm{and} \mathrm{1-3/8} \mathrm{in.} \mathrm{deep.} \mathrm{The} \mathrm{bottom} \mathrm{of}$ this chamber was made of translucent plastic, the back of aluminum, and the top of Bakelite in which were mounted a row of eight equally spaced photocells. Three lights, mounted below the translucent bottom of the chamber, were on during the experiment, and provided light to turn on the photocells. When the rat stuck its nose into the slot one and only one cell was darkened, and an electrical pulse was generated.

Centered at the opposite end of the box, a small metal tray held the 45-mg Noyes rat food pellets delivered by a Gerbrands pellet dispenser. The test stimuli were four click rates delivered at a moderate intensity by a speaker centered behind the slot. The clicks were generated by a Foringer Click Frequency Generator, and had the mean values, in clicks per second, 1.7, 4.6, 10.5, and 24.4. These values are hereafter referred to as stimuli $1,2,3$, and 4 , respectively.

The rats were first adapted to the apparatus, magazine trained, and trained to poke their noses into the slot by reinforcement for a response at any location. A response counted toward reinforcement only if no photocell had been operated during the preceding 0.75 sec. This contingency had, as its main effect, the non-reinforcement of a sweeping movement within the recessed chamber which would operate many photocells in rapid succession.

Responding at each of two locations (photocells numbered 2 and 7) was reinforced in different blocks of sessions, under training stimuli 1 and 4, respectively. Both contingencies were then alternated within experimental sessions. Under the final schedule, in effect for 11 sessions, the stimulus-response contingencies were presented in an irregular order, and appropriate responses were reinforced under a VR 3 requirement.

The generalization test session lasted for $20 \mathrm{~min}$., and consisted of five 1-min. presentations of each of the four stimuli, in an irregular order. The change from one stimulus to the next was immediate, and no reinforcements were delivered during the session.

\section{Results and Discussion}

The mean locations of response for all Ss for the last five sessions of training and during the generalization test are shown in Table 1. (The unit of computation is a photocell position width, rather than inches.)

Table 1. Mean Response Location

\begin{tabular}{lcccc} 
Condition & \multicolumn{4}{c}{ Stimulus } \\
& 1 & 2 & 3 & 4 \\
\hline Training & 2.75 & - & - & 5.38 \\
Test & 4.54 & 4.72 & 5.12 & 5.20 \\
\hline
\end{tabular}



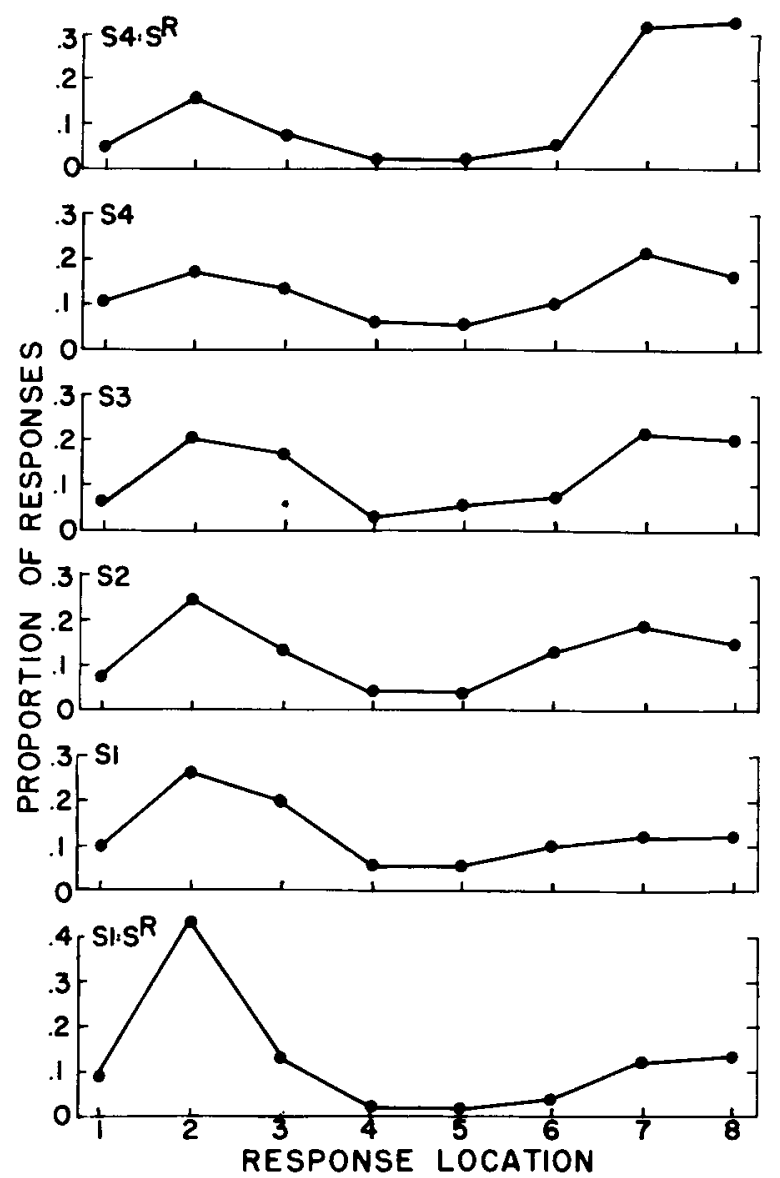

Fig. 1. Proportion of responses at each response location under each of the following conditions (from top to bottom): stimulus 4 , last 5 days of training; stimulus 4 , generalization test; stimulus 3 , test; stimulus 2, test; stimulus 1, test; stimulus 1 , last 5 days of training.

It is clear that, on the average, the rats tended to respond to the appropriate side during training. Also, the mean position of nose insertion varied continuously with changes in click frequency. These pooled results are fairly representative of the individual Ss.

As in the studies previously cited, however, these average results arose not because of a change in the mode of the distribution of nose insertions, but rather because the two responses established during training occurred with varying frequencies during test, giving rise to bimodal distributions. The data for one rat are shown in Fig. 1. The bottom curve is the median response distribution for the last five sessions of training while stimulus 1 was being presented, and the top curve is the corresponding curve during presentations of stimulus 4 . The four other distributions represent responses made during the generalization test. During the test session each stimulus maintained a bimodal distribution of locations, and as click frequency increased, the right hand mode tended to increase, and the left mode, to decrease.

This study, therefore, extends the generality of the studies cited before. Although the stimulus control during training was not as well developed as that in those studies, response distributions during stimulus generalization testing still reflected the bimodality of responding established by training, rather than the continuity of either the stimulus or response dimension. This consistent series of results suggests extension of the principle that responding during stimulus generalization test consists only of those responses established by training, and not of a response amount which reflects the "strength" of a hypothetical continuous response tendency.

\section{References}

Blough, D. S. Interresponse time as a function of continuous variables: a new method and some data. J. exp. Anal. Behav., 1963, 6. 237-246.

Cross, D. V., \& Lane, H. L. On the discriminative control of concurrent responses: the relations among response frequency, latency, and topography in auditory generalization. J. exp. Anal. Behav., 1962, 5, 487-496.

Cumming, W. W., \& Eckerman, D. A. Stimulus control of a differentiated operant. Psychon. Sci., 1965, 3, 313-314.

Migler, B. Effects of averaging data during stimulus generalization. J. exp. Anal. Behav., 1964, 7, 303-307.

\section{Note}

1. This research was supported by USPHS Research Grant MH01604 and the report was prepared, in part, under USPHS Fellowship 1 F3 MH-33,004-01 (PS), from the National Institute of Mental Health. The author thanks R. M. Lee, W. Sloboda, and C. Vogt for help in constructing the apparatus, and $M$. E. Steller for technical assistance. The author's present address is Department of Pharmacology, Royal College of Surgeons of England, Lincoln's Inn Fields, London. W. C. 2. 